\title{
Improvement on the Decay of Crossing Numbers ${ }^{\star}$
}

\author{
Jakub Černý ${ }^{1}$, Jan Kynčl ${ }^{1}$, and Géza Tóth ${ }^{2}$ \\ ${ }^{1}$ Department of Applied Mathematics, Faculty of Mathematics and Physics, Charles \\ University, Malostranské nám. 25, 11800 Praha 1, Czech Republic \\ kuba@kam.mff.cuni.cz, kyncl@kam.mff.cuni.cz \\ 2 Rényi Institute, Hungarian Academy of Sciences, Reáltanoda utca 13-15, H-1053, \\ Budapest, Hungary \\ geza@renyi.hu
}

\begin{abstract}
We prove that the crossing number of a graph decays in a "continuous fashion" in the following sense. For any $\varepsilon>0$ there is a $\delta>0$ such that for $n$ sufficiently large, every graph $G$ with $n$ vertices and $m \geq n^{1+\varepsilon}$ edges has a subgraph $G^{\prime}$ of at most $(1-\delta) m$ edges and crossing number at least $(1-\varepsilon) \mathrm{CR}(G)$. This generalizes the result of J. Fox and Cs. Tóth.
\end{abstract}

\section{Introduction}

For any graph $G$, let $n(G)$ (resp. $m(G)$ ) denote the number of its vertices (resp. edges). If it is clear from the context, we simply write $n$ and $m$ instead of $n(G)$ and $m(G)$. The crossing number $\mathrm{CR}(G)$ of a graph $G$ is the minimum number of edge crossings over all drawings of $G$ in the plane. In the optimal drawing of $G$, crossings are not necessarily distributed uniformly among the edges. Some edges could be more "responsible" for the crossing number than some other edges. For any fixed $k$, it is not hard to construct a graph $G$ whose crossing number is $k$, but $G$ has an edge $e$ such that $G \backslash e$ is planar. Richter and Thomassen RT93. started to investigate the following general problem. We have a graph $G$, and we want to remove a given number of edges. By at least how much does the crossing number decrease? They conjectured that there is a constant $c$ such that every graph $G$ with $\operatorname{CR}(G)=k$ has an edge $e$ with $\operatorname{CR}(G \backslash e) \geq k-c \sqrt{k}$. They only proved that $G$ has an edge with $\mathrm{CR}(G \backslash e) \geq \frac{2}{5} \mathrm{CR}(G)-O(1)$.

Pach, Radoičić, Tardos, and Tóth PRTT06 proved that for every graph $G$ with $m(G) \geq \frac{103}{16} n(G)$, we have $\mathrm{CR}(G) \geq 0.032 \frac{m^{3}}{n^{2}}$. It is not hard to see PT00. that for any edge $e$, we have $\mathrm{CR}(G-e) \geq \mathrm{CR}(G)-m+1$. These two results imply

\footnotetext{
* Jakub Černý and Jan Kynčl have been supported by the Phenomena in High Dimensions project, in the framework of the European Community's "Structuring the European Research Area" programme, Grant MRTN-CT-2004-511953 at Rényi Institute and by project 1M0021620808 (ITI) of Ministry of Education of the Czech Republic; Géza Tóth has been supported by the Hungarian Research Fund grants OTKA-T-038397, OTKA-T-046246, and OTKA-K-60427.
} 
an improvement of Richter-Thomassen bound if $m \geq 8.1 n$, and also imply the Richter-Thomassen conjecture for graphs of $\Omega\left(n^{2}\right)$ edges.

J. Fox and Cs. Tóth FT06 investigated the case where we want to delete a positive fraction of the edges.

Theorem A. FT06] For every $\varepsilon>0$, there is an $n_{\varepsilon}$ such that every graph $G$ with $n(G) \geq n_{\varepsilon}$ vertices and $m(G) \geq n(G)^{1+\varepsilon}$ edges has a subgraph $G^{\prime}$ with

$$
m\left(G^{\prime}\right) \leq\left(1-\frac{\varepsilon}{24}\right) m(G)
$$

and

$$
\mathrm{CR}\left(G^{\prime}\right) \geq\left(\frac{1}{28}-o(1)\right) \mathrm{CR}(G)
$$

In this note we generalize Theorem A.

Theorem. For every $\varepsilon, \gamma>0$, there is an $n_{\varepsilon, \gamma}$ such that every graph $G$ with $n(G) \geq n_{\varepsilon, \gamma}$ vertices and $m(G) \geq n(G)^{1+\varepsilon}$ edges has a subgraph $G^{\prime}$ with

$$
m\left(G^{\prime}\right) \leq\left(1-\frac{\varepsilon \gamma}{2394}\right) m(G)
$$

and

$$
\mathrm{CR}\left(G^{\prime}\right) \geq(1-\gamma) \mathrm{CR}(G)
$$

\section{Proof of the Theorem}

Our proof is based on the argument of Fox and Tóth [FT06], the only new ingredient is Lemma 1 .

Definition. Let $r \geq 2, p \geq 1$ be integers. A $2 r$-earring of size $p$ is a graph which is a union of an edge $u v$ and $p$ edge-disjoint paths between $u$ and $v$, each of length at most $2 r-1$. Edge $u v$ is called the main edge of the $2 r$-earring.

Lemma 1. Let $r \geq 2, p \geq 1$ be integers. There exists $n_{0}$ such that every graph $G$ with $n \geq n_{0}$ vertices and $m \geq 6 \mathrm{prn}^{1+1 / r}$ edges contains at least $m / 3 \mathrm{pr}$ edgedisjoint $2 r$-earrings, each of size $p$.

Proof. By the result of Alon, Hoory, and Linial AHL02, for some $n_{0}$, every graph with $n \geq n_{0}$ vertices and at least $n^{1+1 / r}$ edges contains a cycle of length at most $2 r$.

Suppose that $G$ has $n \geq n_{0}$ vertices and $m \geq 6 p r n^{1+1 / r}$ edges. Take a maximal edge-disjoint set $\left\{E_{1}, E_{2}, \ldots, E_{x}\right\}$ of $2 r$-earrings, each of size $p$. Let $E=E_{1} \cup$ $E_{2} \cup \cdots \cup E_{x}$, the set of all edges of the earrings and let $G^{\prime}=G \backslash E$. Now let $E_{1}^{\prime}$ be a $2 r$-earring of $G^{\prime}$ of maximum size. Note that this size is less than $p$. Let $G_{1}^{\prime}=G^{\prime} \backslash E_{1}^{\prime}$. Similarly, let $E_{2}^{\prime}$ be a $2 r$-earring of $G_{1}^{\prime}$ of maximum size and let $G_{2}^{\prime}=G_{1}^{\prime} \backslash E_{2}^{\prime}$. Continue analogously, as long as there is a $2 r$-earring in the 
remaining graph. We obtain the $2 r$-earrings $E_{1}^{\prime}, E_{2}^{\prime}, \ldots, E_{y}^{\prime}$, and the remaining graph $G^{\prime \prime}=G_{y}^{\prime}$ does not contain any $2 r$-earring. Let $E^{\prime}=E_{1}^{\prime} \cup E_{2}^{\prime} \cup \cdots \cup E_{y}^{\prime}$.

We claim that $y<n^{1+1 / r}$. Suppose on the contrary that $y \geq n^{1+1 / r}$. Take the main edges of $E_{1}^{\prime}, E_{2}^{\prime}, \ldots, E_{y}^{\prime}$. We have at least $n^{1+1 / r}$ edges so by the result of Alon, Hoory, and Linial AHL02 some of them form a cycle $C$ of length at most $2 r$. Let $i$ be the smallest index with the property that $C$ contains the main edge of $E_{i}^{\prime}$. Then $C$, together with $E_{i}^{\prime}$ would be a $2 r$-earring of $G_{i-1}^{\prime}$ of greater size than $E_{i}^{\prime}$, contradicting the maximality of $E_{i}^{\prime}$.

Each of the earrings $E_{1}^{\prime}, E_{2}^{\prime}, \ldots, E_{y}^{\prime}$ has at most $(p-1)(2 r-1)+1$ edges so we have $\left|E^{\prime}\right| \leq y(p-1)(2 r-1)+y<(2 p r-1) n^{1+1 / r}$. The remaining graph, $G^{\prime \prime}$ does not contain any $2 r$-earring, in particular, it does not contain any cycle of length at most $2 r$, since it is a $2 r$-earring of size one. Therefore, by AHL02, for the number of its edges we have $e\left(G^{\prime \prime}\right)<n^{1+1 / r}$.

It follows that the set $E=\left\{E_{1}, E_{2}, \ldots, E_{x}\right\}$ contains at least $m-2 p r n^{1+1 / r} \geq$ $\frac{2}{3} m$ edges. Each of $E_{1}, E_{2}, \ldots, E_{x}$ has at most $p(2 r-1)+1 \leq 2 p r$ edges, therefore, $x \geq m / 3 p r$.

Lemma 2. FT06 Let $G$ be a graph with $n$ vertices, $m$ edges, and degree sequence $d_{1} \leq d_{2} \leq \cdots \leq d_{n}$. Let $\ell$ be the integer such that $\sum_{i=1}^{\ell-1} d_{i}<4 m / 3$ but $\sum_{i=1}^{\ell} d_{i} \geq 4 m / 3$. If $n$ is large enough and $m=\Omega\left(n \log ^{2} n\right)$ then

$$
\mathrm{CR}(G) \geq \frac{1}{65} \sum_{i=1}^{\ell} d_{i}^{2} .
$$

Proof of the Theorem. Let $\varepsilon, \gamma \in(0,1)$ be fixed. Choose integers $r, p$ such that $\frac{1}{r}<\varepsilon \leq \frac{2}{r}$ and $\frac{132}{p}<\gamma \leq \frac{133}{p}$. Then there is an $n_{\varepsilon, \gamma}$ with the following properties: (a) $n_{\varepsilon, \gamma} \geq n_{0}$ from Lemma 1 (b) $\left(n_{\varepsilon, \gamma}\right)^{1+\varepsilon}>6 p r \cdot\left(n_{\varepsilon, \gamma}\right)^{1+1 / r}$,

Let $G$ be a graph with $n \geq n_{\varepsilon, \gamma}$ vertices and $m \geq n^{1+\varepsilon}$ edges.

Let $v_{1}, \ldots, v_{n}$ be the vertices of $G$, of degrees $d_{1} \leq d_{2} \leq \cdots \leq d_{n}$ and define $\ell$ as in Lemma 2, that is, $\sum_{i=1}^{\ell-1} d_{i}<4 m / 3$ but $\sum_{i=1}^{\ell} d_{i} \geq 4 m / 3$. Let $G_{0}$ be the subgraph of $G$ induced by $v_{1}, \ldots, v_{\ell}$. Observe that $G_{0}$ has at least $m / 3$ edges. Therefore, by Lemma 1 G $G_{0}$ contains at least $m / 9 p r$ edge-disjoint $2 r$-earrings, each of size $p$.

Let $M$ be the set of the main edges of these $2 r$-earrings. We have $|M| \geq$ $m / 9 p r \geq \frac{\varepsilon \gamma}{2394} m$. Let $G^{\prime}=G \backslash M$ and $G_{0}^{\prime}=G_{0} \backslash M$.

Take an optimal drawing $D\left(G^{\prime}\right)$ of the subgraph $G^{\prime} \subset G$. We have to draw the missing edges to obtain a drawing of $G$. Our method is a randomized variation of the embedding method, which has been applied by Leighton [L83], Richter and Thomassen [RT93, Shahrokhi et al. SSSV97, Székely S04, and most recently by Fox and Tóth [FT06]. For every missing edge $e_{i}=u_{i} v_{i} \in M \subset G_{0}, e_{i}$ is the deleted main edge of a $2 r$-earring $E_{i} \subset G_{0}$. So there are $p$ vertex-disjoint paths in $G_{0}$ from $u_{i}$ to $v_{i}$. For each of these paths, draw a curve from $u_{i}$ to $v_{i}$ infinitesimally close to that path. Call these $p$ curves potential $u_{i} v_{i}-e d g e s$ and call the resulting drawing $D$. 
To get a drawing of $G$, for each $e_{i}=u_{i} v_{i} \in M$, choose one of the $p$ potential $u_{i} v_{i}$-edges at random, independently and uniformly, with probability $1 / p$, and draw the edge $u_{i} v_{i}$ as that curve.

There are two types of new crossings in the obtained drawing of $G$. First category crossings are infinitesimally close to a crossing in $D\left(G^{\prime}\right)$, second category crossings are infinitesimally close to a vertex of $G_{0}$ in $D\left(G^{\prime}\right)$.

The expected number of first category crossings is at most

$$
\left(1+\frac{2}{p}+\frac{1}{p^{2}}\right) \mathrm{CR}\left(G^{\prime}\right)=\left(1+\frac{1}{p}\right)^{2} \mathrm{CR}\left(G^{\prime}\right) .
$$

Indeed, for each edge of $G^{\prime}$, there can be at most one new edge drawn next to it, and that is drawn with probability at most $1 / p$. Therefore, in the close neighborhood of a crossing in $D\left(G^{\prime}\right)$, the expected number of crossings is at most $\left(1+\frac{2}{p}+\frac{1}{p^{2}}\right)$. See figure 1(a).

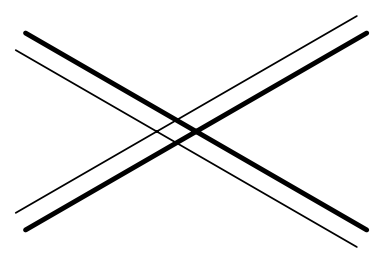

(a)

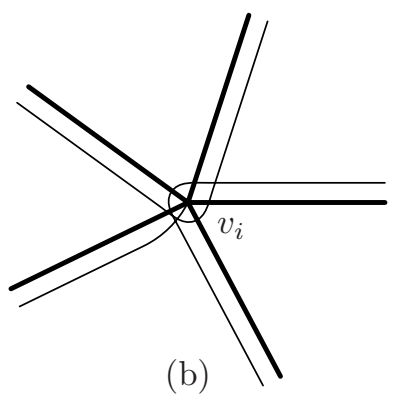

(b)

Fig. 1. The thick edges are edges of $G^{\prime}$, the thin edges are the potential edges. Figure shows (a) a neighborhood of a crossing in $D\left(G^{\prime}\right)$ and (b) a neighborhood of a vertex $v_{i}$ in $G^{\prime}$.

In order to estimate the expected number of second category crossings, consider the drawing $D$ near a vertex $v_{i}$ of $G_{0}$. In the neighborhood of vertex $v_{i}$ we have at most $d_{i}$ original edges. Since we draw at most one potential edge along each original edge, there can be at most $d_{i}$ potential edges in the neighborhood. Each potential edge can cross each original edge at most once, and any two potential edges can cross at most twice. See figure1(b). Therefore, the total number of first category crossings in $D$ in the neighborhood of $v_{i}$ is at most $2 d_{i}^{2}$. (This bound can be substantially improved with a more careful argument, see e. g. [FT06, but we do not need anything better here.) To obtain the drawing of $G$, we keep each of the potential edges with probability $1 / p$, so the expected number of crossings in the neighborhood of $v_{i}$ is at most $\frac{1}{p} 2 d_{i}^{2}$.

Therefore, the total expected number of crossings in the random drawing of $G$ is at most $\left(1+\frac{2}{p}+\frac{1}{p^{2}}\right) \mathrm{CR}\left(G^{\prime}\right)+\frac{2}{p} \sum_{i=1}^{\ell} d_{i}^{2}$.

There exists an embedding with at most this many crossings, therefore, by Lemma 2 we have 


$$
\mathrm{CR}(G) \leq\left(1+\frac{1}{p}\right)^{2} \mathrm{CR}\left(G^{\prime}\right)+\frac{2}{p} \sum_{i=1}^{\ell} d_{i}^{2} \leq\left(1+\frac{1}{p}\right)^{2} \mathrm{CR}\left(G^{\prime}\right)+\frac{130}{p} \mathrm{CR}(G) .
$$

It follows that

$$
\left(1-\frac{130}{p}\right) \mathrm{CR}(G) \leq\left(1+\frac{1}{p}\right)^{2} \mathrm{CR}\left(G^{\prime}\right)
$$

So

$$
\left(1-\frac{130}{p}\right)\left(1-\frac{1}{p}\right)^{2} \mathrm{CR}(G) \leq \mathrm{CR}\left(G^{\prime}\right)
$$

consequently

$$
\mathrm{CR}\left(G^{\prime}\right) \geq\left(1-\frac{132}{p}\right) \mathrm{CR}(G) \geq(1-\gamma) \mathrm{CR}(G) .
$$

Remark. In the statement of our Theorem we cannot require that every subgraph $G^{\prime}$ with $(1-\delta) m(G)$ edges has crossing number $\operatorname{CR}\left(G^{\prime}\right) \geq(1-\gamma) \mathrm{CR}(G)$, instead of just one such subgraph $G^{\prime}$. In fact, the following statement holds.

Proposition. For every $\varepsilon \in(0,1)$ there exist graphs $G_{n}$ with $n\left(G_{n}\right)=\Theta(n)$ vertices and $m\left(G_{n}\right)=\Theta\left(n^{1+\varepsilon}\right)$ edges with subgraphs $G_{n}^{\prime} \subset G_{n}$ such that

$$
m\left(G_{n}^{\prime}\right)=(1-o(1)) m\left(G_{n}\right)
$$

and

$$
\operatorname{CR}\left(G_{n}^{\prime}\right)=o\left(\operatorname{CR}\left(G_{n}\right)\right)
$$

Proof. Roughly speaking, $G_{n}$ will be the disjoint union of a large graph $G_{n}^{\prime}$ with low crossing number and a small graph $H_{n}$ with large crossing number. More precisely, let $G=G_{n}$ be a disjoint union of graphs $G^{\prime}=G_{n}^{\prime}$ and $H=H_{n}$, where $G^{\prime}$ is a disjoint union of $\Theta\left(n^{1-\varepsilon}\right)$ complete graphs, each with $n^{\varepsilon}$ vertices and $H$ is a complete graph with $n^{(3+5 \varepsilon) / 8}$ vertices. We have $m(G)=\Theta\left(n^{1+\varepsilon}\right)$ and $m(H)=\Theta\left(n^{(3+5 \varepsilon) / 4}\right)=o(m(G))$, since $\frac{3+5 \varepsilon}{4}<1+\varepsilon$. By the crossing lemma (see e. g. [PRTT06] $), \operatorname{CR}(G) \geq \mathrm{CR}(H)=\Omega\left(n^{(3+5 \varepsilon) / 2}\right)$, but $\mathrm{CR}\left(G^{\prime}\right)=O\left(n^{1-\varepsilon} \cdot n^{4 \varepsilon}\right)=$ $O\left(n^{1+3 \varepsilon}\right)=o(\mathrm{CR}(G))$, because $\frac{3+5 \varepsilon}{2}>1+3 \varepsilon$.

On the other hand, we conjecture that we can require that a positive fraction of all subgraphs $G^{\prime}$ of $G$ with $(1-\delta) m(G)$ edges has crossing number $\operatorname{CR}\left(G^{\prime}\right) \geq$ $(1-\gamma) \mathrm{CR}(G)$. The simplest form of our conjecture is the following.

Conjecture. For every $\varepsilon>0$, there is an $n_{\varepsilon}$ and $\delta$ such that every graph $G$ with $n(G) \geq n_{\varepsilon}$ vertices and $m(G) \geq n(G)^{1+\varepsilon}$ edges has the following property. Let $G^{\prime}$ be a random subgraph of of $G$ such that we choose each edge of $G$ independently with probability $p=1-\delta$. Then

$$
\operatorname{Pr}\left[\mathrm{CR}\left(G^{\prime}\right) \geq(1-\varepsilon) \mathrm{CR}(G)\right]>\delta .
$$




\section{References}

[AHL02] Alon, N., Hoory, S., Linial, N.: The Moore bound for irregular graphs. Graphs and Combinatorics 18, 53-57 (2002)

[FT06] Fox, J., Tóth, Cs.: On the decay of crossing numbers. In: Kaufmann, M., Wagner, D. (eds.) GD 2006. LNCS, vol. 4372, pp. 174-183. Springer, Heidelberg (2007)

[L83] Leighton, T.: Complexity issues in VLSI. MIT Press, Cambridge (1983)

[PRTT06] Pach, J., Radoičić, R., Tardos, G., Tóth, G.: Improving the Crossing Lemma by finding more crossings in sparse graphs. In: Proc. 19th ACM Symposium on Computational Geometry, pp. 68-75 (2004), Also in: Discrete and Computational Geometry 36, 527-552 (2006)

[PT00] Pach, J., Tóth, G.: Thirteen problems on crossing numbers. Geombinatorics 9, 194-207 (2000)

[RT93] Richter, B., Thomassen, C.: Minimal graphs with crossing number at least $k$. J. Combin. Theory Ser. B 58, 217-224 (1993)

[SSSV97] Shahrokhi, F., Sýkora, O., Székely, L., Vrt'o, I.: Crossing numbers: bounds and applications. In: Intuitive geometry, Budapest (1995) Bolyai Soc. Math. Stud. 6, 179-206 (1997)

[S04] Székely, L.: Short proof for a theorem of Pach, Spencer, and Tóth. In: Towards a theory of geometric graphs, Contemporary Mathematics, vol. 342, pp. 281-283. AMS, Providence, RI (2004) 